\title{
Publisher Correction: An investigation of children's strategies for overcoming the tragedy of the commons
}

Rebecca Koomen (D) and Esther Herrmann

Correction to: Nature Human Behaviour https://doi.org/10.1038/s41562-018-0327-2, published online 16 April 2018.

In the version of this Article originally published, two notes reading '[CJ1]' and '[CJ1]This should stay the same. It does refer to a particular seminal experiment and is also not the subject of the citation (40) which refers to a review article' were mistakenly left in the seventh paragraph of the text. These notes have now been removed.

Published online: 21 June 2018

https://doi.org/10.1038/s41562-018-0367-7 